\title{
Evaluation of the Multilook Size in Polarimetric Optimization of Differential SAR Interferograms
}

\author{
Alejandro Mestre-Quereda, Student Member, IEEE, Juan. M Lopez-Sanchez, Senior Member, IEEE, J. David \\ Ballester-Berman, Pablo J. Gonzalez, Andrew Hooper, Senior Member, IEEE, and Tim J. Wright
}

\begin{abstract}
The interferometric coherence is a measure of the correlation between two SAR images and constitutes a commonlyused estimator of the phase quality. Its estimation requires a spatial average within a two-dimension window, usually named as multilook. The multilook processing allows to reduce noise at the expenses of a resolution loss. In this work, we analyze the influence of the multilook size while applying a polarimetric optimization of the coherence. The same optimization algorithm has been carried out with different multilook sizes and also with the NL-SAR filter, which has the advantage of preserving the original resolution of the interferogram. Our experiments have been carried out with a single pair of quad-polarimetric RADARSAT-2 images mapping the Mount Etna's volcanic eruption of May 2008. Results obtained with this particular dataset show that the coherence is increased notably with respect to conventional channels when small multilook sizes are employed, especially over low-vegetated areas. Conversely, very decorrelated areas benefit from larger multilook sizes but do not exhibit an additional improvement with the polarimetric optimization.
\end{abstract}

Index Terms-Differential SAR interferometry, phase noise, polarimetry, optimization, filtering

\section{INTRODUCTION}

D IFFERENTIAL SAR interferometry (DInSAR) is a remote sensing technique employed to monitor surface displacements, such as the ground subsidence or uplift and the deformation associated with geological events (eartquakes and volcanic eruptions) [1], [2]. Historically, DInSAR techniques have been applied with a single polarimetric channel due to the lack of multi-polarization SAR data. However, as newer SAR satellites, for instance, ALOS-1/2, RADARSAT-2 or TerraSAR-X are able to acquire polarimetric data, ground deformation quality results could be improved with the inclusion of polarimetric information.

In previous works with multitemporal data sets (i.e. time series), the benefits of processing all the available polarimetric

This work was supported by the Spanish Ministry of Economy, Industry and Competitiveness (MINECO), the State Agency of Research (AEI) and the European Funds for Regional Development (EFRD) under Projects TIN201455413-C2-2-P and TEC2017-85244-C2-1-P, by the Spanish Ministry of Education under grant PRX14/00151, and by the UK Natural Environmental Research Council (NERC) through the Centre for the Observation and Modelling of Earthquakes, Volcanoes and Tectonics (COMET, GA/13/M/031).

A. Mestre-Quereda, J. M. Lopez-Sanchez and J. D. Ballester-Berman are with the Signals, Systems and Telecommunications Group, IUII, University of Alicante, Alicante, E-03080, Spain (e-mail: alejandro.mestre@ua.es, juanma.lopez@ua.es,davidb@ua.es).

Pablo J. Gonzalez is with COMET and Dept. of Earth, Ocean and Ecological Sciences. School of Environmental Sciences, University of Liverpool, United Kingdom (e-mail: pjgonzal@ liverpool.ac.uk)

A. Hooper and T. Wright are with COMET, School of Earth and Environment, University of Leeds, Leeds, U.K. (e-mail: a.hooper@leeds.ac.uk, t.j.wright@leeds.ac.uk) information were proved, as detailed in [3]-[7]. These polarimetric DInSAR (PolDInSAR) methods improve the quality of the final results, in terms of number of valid pixels and deformation measurement accuracy, compared to time series of single-polarimetric DInSAR. However, PolDInSAR processing methods are still to be tested in applications based on a single interferogram, so a first study is presented in this work. As it is well-known, the interferometric coherence constitutes a direct indicator of the phase quality [8], since it quantifies the correlation between the two SAR images combined. In practice, coherence estimation requires a spatial average inside an estimation window of a certain size. This average operation is usually named as multilook, and it is known to modify signal statistics and reduce the so-called speckle effect, at the expenses of a spatial resolution loss [9], [10].

In this work, we have analyzed the effect of the multilook size in coherence optimization for PolDInSAR with a single interferogram, for which we have applied a polarimetric optimization varying the size of the estimation window, i.e. the multilook size: from $3 \times 3$ to $21 \times 21$ independent sample averages. Additionally, the Non-local SAR filter (NL-SAR) [11] has also been tested. This filter has the advantage of effectively reduce noise while preserving when necessary the spatial resolution of the original images, as non-contiguous pixels are averaged according to a similarity criterion.

Different polarimetric optimization algorithms are available in the literature [6], [7], being the Exhaustive Search Polarimetric Optimization (ESPO) the one providing the best result, at the expenses of a high computational cost. In this work, a recently published method has been applied, known as improved Signal-to-Noise ratio (SNR) optimization (IM-SNROPT) [12]. This method divides the 4-D optimization problem imposed by ESPO into three independent and successive optimizations in 1-D, 1-D and 2-D [12]. Then, the computational burden is greatly reduced and the final solution is very similar to ESPO.

\section{Polarimetric Optimization}

The basis of the polarimetric optimization of coherence relies on the general concept of polarimetric SAR interferometry (PolInSAR), introduced in [13]. For quad-polarimetric SAR data, the $2 \times 2$ scattering matrix $[S]$ is used to obtain the target vector $k$ by a projection onto a group of orthogonal matrices. Using the Pauli basis, the target vector is defined as

$$
k=\frac{1}{\sqrt{2}}\left[S_{h h}+S_{v v}, S_{h h}-S_{v v}, 2 S_{h v}\right]^{T},
$$


where $H H$ and $V V$ are the horizontal and vertical copolar channels respectively, $H V$ is the crosspolar channel, and we assume $H V=V H$ because of the reciprocity theorem, which implies the scattering matrix $[S]$ to be symmetric. In order to generate an interferogram, PolInSAR techniques work by projecting the complex target vector $k$ using a unitary complex vector $\omega$, resulting in a complex scattering coefficient $\mu=$ $\omega^{* \mathrm{~T}} k$ [13], where ${ }^{* \mathrm{~T}}$ is the Hermitian transpose operation. Thus, as in the single-polarization case, we can apply to $\mu$ any DInSAR processing technique [6].

The objective of the optimization is to enhance the differential phase quality by exploring the whole polarimetric space. To this aim, vector $\omega$ is chosen to optimize a suitable cost function or quality criterion. Different quality estimators have been considered in this work. The first one is the conventional interferometric coherence, defined as [13]

$$
|\gamma|=\frac{\left|\omega_{1}^{* T} \Omega_{12} \omega_{2}\right|}{\sqrt{\omega_{1}^{* T} T_{11} \omega_{1}} \sqrt{\omega_{2}^{* T} T_{22} \omega_{2}}}
$$

where $T_{11}$ and $T_{22}$ are the polarimetric coherency matrices of master and slave images, and $\Omega_{12}$ is the polarimetric interferometric cross-correlation matrix defined in [13]:

$$
\begin{aligned}
& T_{11}=E\left\{k_{1} k_{1}^{* \mathrm{~T}}\right\} \\
& T_{22}=E\left\{k_{2} k_{2}^{* \mathrm{~T}}\right\} \\
& \Omega_{12}=E\left\{k_{1} k_{2}^{* \mathrm{~T}}\right\}
\end{aligned}
$$

where $E\{\}$ denotes the spatial average needed in the estimation, i.e. the multilook.

Additionally, the interferometric phase $\phi$ is

$$
\phi=\arg \left(\omega^{* \mathrm{~T}}\left[\Omega_{12}\right] \omega\right) .
$$

From (4), we can derive a second quality estimator which corresponds to the local correlation based only on interferometric phase values. Such an estimator, called phase coherence, also requires a spatial average around a pixel's neighborhood. Its expression is

$$
\gamma_{p h}=\frac{1}{M} \sqrt{\sum_{m=0}^{M-1} \cos ^{2}\left(\phi_{m}\right)+\sum_{m=0}^{M-1} \sin ^{2}\left(\phi_{m}\right)}
$$

where $M$ is the number of averaged samples (pixels).

The last quality criterion considered in this work is the phase standard deviation in a area. The phase standard deviation provides a meaningful measure of the phase noise, and it can be directly estimated from the interferogram phase values, provided that the set of pixels belongs to a homogeneous area.

\section{DATA SET}

We have used a set of two quad-pol single-look complex (SLC) images acquired by the RADARSAT-2 satellite over the Mount Etna's (Italy) eruption in May 2008. Images were acquired in 2008-05-05 and 2008-05-29, using FQ29 beam mode, which near and far incidence angles are $46.8^{\circ}$ and $48.0^{\circ}$, respectively. The processed image size is $3700 \times 6000$ pixels. A Pauli RGB composite image of the studied area is shown in Fig. 1. We have also selected four different regions of interest (ROI), delimited by the square polygons in Fig. 1, to be analyzed in detail later, which correspond to different land-cover types: a rural area, an area with vegetation, an area without vegetation (i.e. a bare surface), and a zone with changes since it was altered by the lava flow after the volcanic eruption. The size of each ROI is $200 \times 200$ pixels.

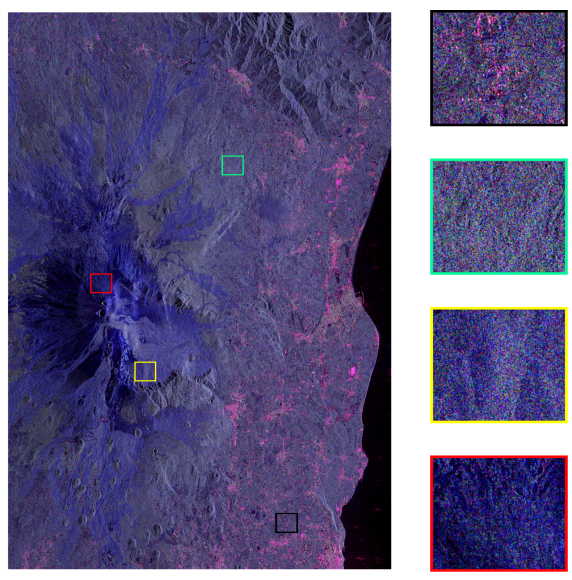

Fig. 1. Composite RGB image of the area under study. $\mathrm{R}=\mathrm{HH}-\mathrm{VV}, \mathrm{G}=$ $2 \mathrm{HV}, \mathrm{B}=\mathrm{HH}+\mathrm{VV}$. Different regions of interest: rural area (black square), area with vegetation (green square), area without vegetation (yellow square), and changed area (red square).

\section{RESULTS}

\section{A. Coherence Optimization}

Coherence optimization consists in finding the optimum combination of polarimetric channels that maximizes $|\gamma|$ defined in (2). As stated before, a spatial average is needed in order to estimate $|\gamma|$, but this is a biased estimator [8]: overestimation (positive bias) occurs when small windows are employed, that is, the obtained coherence values are larger than the true values due to the small number of averaged pixels. On the contrary, the use of larger windows provides better estimates (less biased) but it entails the risk of mixing nonhomogeneous pixels inside the averaging window, making the estimation less reliable and useful.

In order to analyze the influence of the size of the estimation window in the optimization, coherence is estimated with a boxcar filter which size varies from $3 \times 3$ to $21 \times 21$. The input data in all cases are the SLC images, without any previous multilooking. In addition, the NL-SAR filter has been also tested. In this case, resolution is better preserved as only statistically homogeneous pixels (not necessarily contiguous) are averaged. Consequently, structure details are not blurred and strong scatterers with high coherence values are not mixed with surrounding pixels with a different response. Concerning the NL-SAR filtering parameters, a search window size of $25 \times 25$ pixels and a patch size of $5 \times 5$ pixels have been used. Finally, the optimization is carried out in all cases at every multilooked pixel for the full scene represented in Fig. 1.

In Fig. 2 we show the coherence maps of the optimum channels for different number of looks. For comparison purposes, we also show the coherence maps of the 1st channel in the Pauli basis, i.e. HH+VV. Comparing the two rows of coherence maps, we observe that coherence is globally increased 
with the polarimetric optimization, especially with a $3 \times 3$ multilook. In that case, an overestimation of the coherence associated with the small number of samples averaged was expected [8] (hence the higher coherences in both Figs. 2(a) and (e)). The largest increase of coherence is obtained with this multilook size. The mean coherence of the whole scene is increased by 0.31 (from 0.53 to 0.84 ). The positively biased estimator makes coherence to be increased even in areas where coherence should be null theoretically, such as over the sea (right part of the image). In addition, it must be stated that the bias in coherence estimation is increased when we combine two or more polarimetric channels to optimize the coherence, since the additional degrees of freedom allow us a mathematical maximization of the coherence, regardless of the scattering physics present in the scene.

As the multilook size increases, the initial values of estimated coherence decrease, as shown in Fig. 2(b) and (c). This is due to two factors: less bias in coherence estimation, and mixing of non-homogeneous pixels in the averaging window. Regarding the optimum values, there is an inability to find a polarimetric combination which response is significantly better than others, making the final improvement less evident in comparison with smaller multilook sizes. For instance, with a multilook size of $19 \times 19$ pixels, the mean coherence in the scene is only increased by 0.09 (from 0.33 to 0.42 ). The average increase of coherence for all multilook sizes is represented in Fig. 4(a), showing that the coherence improvement is inversely proportional to the multilook size.

Concerning the optimization applied to the non-local filtered data, the improvement in the coherence is quite remarkable, as shown in Fig. 2(h). coherence is greatly increased in the whole interferogram (the mean coherence of the scene increases by 0.20 ), especially in relevant areas related to surface deformation around the volcano crater and in urban areas. However, in this case, it is not much overestimated in highly decorrelated zones, as it was obtained with a $3 \times 3$ multilook. For instance, coherence is not increased notably on the sea area of the right side of the image. This fact is in line with resolution preservation and with the mixing of only homogeneous pixels. As in most adaptive filters, the resulting equivalent number of looks after using the NL-SAR filter is variable over the scene, so the previously mentioned estimation bias and the additional bias generated by the polarimetric optimization should be considered carefully in the interpretation of this result.

The coherence increase is also illustrated in Fig. 3 with the histograms of $|\gamma|$ of all polarimetric channels. The largest increase is clearly observed when coherence is estimated with small windows especially with $3 \times 3$ ). Pixels that originally had a very low coherence (between 0 and 0.4 ) now present coherences distributed from 0.45 to 0.9 in the optimum channel. Moreover, pixels with high coherences in any polarimetric channel (greater than 0.8) also have their values increased in the optimum case. As previously stated, this increase is larger with a $3 \times 3$ multilook, and decreases progressively. In fact, for larger estimation windows, there is only a small improvement in comparison to channel $\mathrm{HH}+\mathrm{VV}$, as shown especially in Fig. 3(c) for high coherence values. More specifically, with a $3 \times 3$ multilook, $69.3 \%$ of pixels exhibit coherences greater than 0.8 in the optimum channel, whereas this percentage is only $17.9 \%$ in the $\mathrm{HH}+\mathrm{VV}$ channel. However, in the $9 \times 9$ case, $16.3 \%$ of pixels have coherence values greater than 0.8 in the optimum channel, against $12.4 \%$ in the $\mathrm{HH}+\mathrm{VV}$ channel (difference of just $3.9 \%$ ).
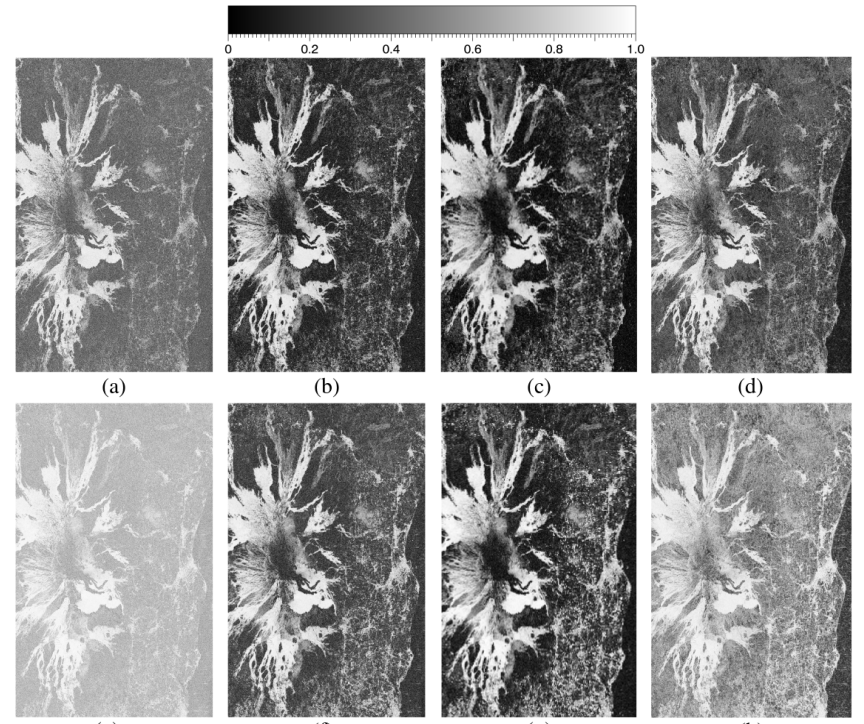

(f)
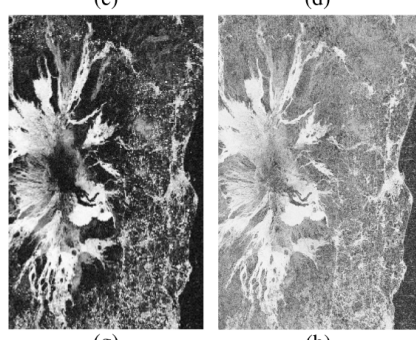

Fig. 2. Coherence maps of channels $\mathrm{HH}+\mathrm{VV}$ (top) and computed optimum (bottom) for different number of looks: (a)-(e) $3 \times 3$. (b)-(f) $9 \times 9$. (c)(g) $19 \times 19$. (d)-(h) NL-SAR.

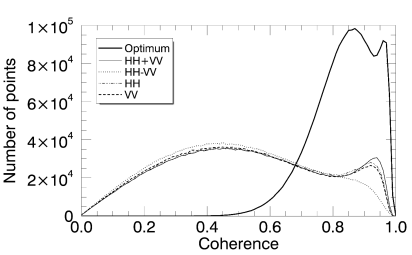

(a)

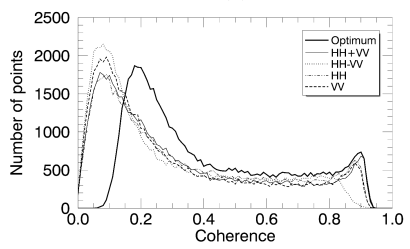

(c)

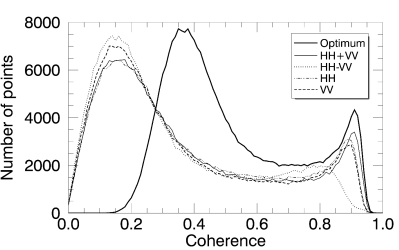

(b)

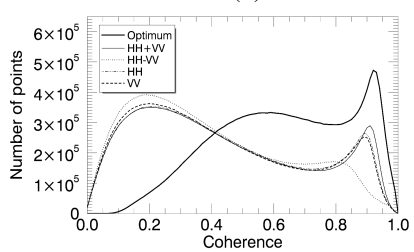

(d)
Fig. 3. Histograms of estimated coherence for different polarimetric channels $(\mathrm{HH}, \mathrm{VV}, \mathrm{HH}+\mathrm{VV}, \mathrm{HH}-\mathrm{VV})$ and optimum channels for different number of looks: (a) $3 \times 3$. (b) $9 \times 9$. (c) $19 \times 19$. (d) NL-SAR.

The second cost function that has been optimized is the phase coherence, which can be estimated with (4) and (5). The histograms of $\gamma_{p h}$ for three different number of looks and for different polarimetric channels are represented in Fig. 5. The optimization provides the same results as in the previous case: a major improvement for small estimation windows which decreases when large estimation windows are used. Additionally, in Fig. 4(b) we show the mean phase coherence differences between the optimum channel and $\mathrm{HH}+\mathrm{VV}$ channel. As in the $|\gamma|$ optimization case, the larger improvement is obtained with small multilook sizes and decreases progressively. 


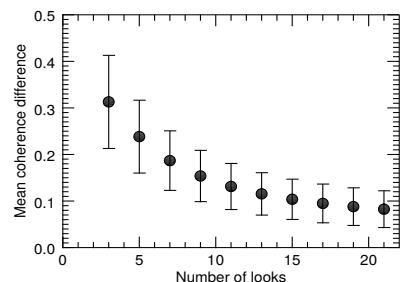

(a)

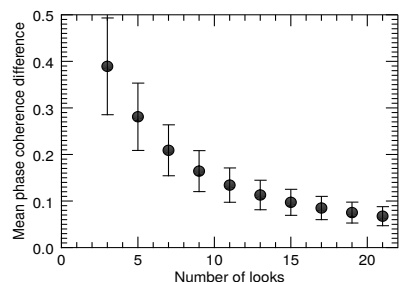

(b)
Fig. 4. Average difference between the coherence (a) and the phase coherence (b) computed at the optimum channel and at $\mathrm{HH}+\mathrm{VV}$ channel for different number of looks (from $3 \times 3$ to $21 \times 21$ ).

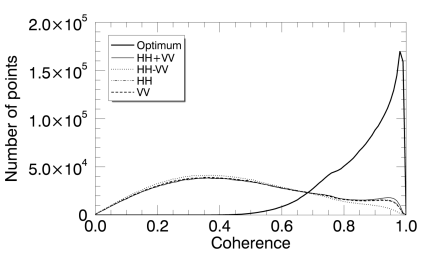

(a)

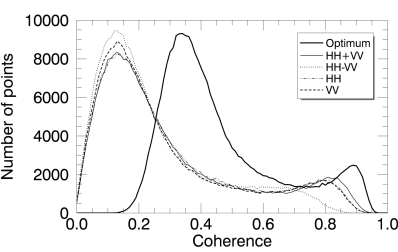

(b)

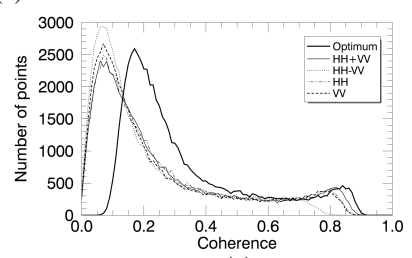

(c)

Fig. 5. Histograms of estimated phase coherence for different polarimetric channels, $\mathrm{HH}, \mathrm{VV}, \mathrm{HH}+\mathrm{VV}, \mathrm{HH}-\mathrm{VV}$ and optimum channels. (a) $3 \times 3$. (b) $9 \times 9$. (c) $19 \times 19$.

Besides coherence, we need to check the phase improvement obtained at the different optimization cases. The differential phases of the optimum channel and the $\mathrm{HH}+\mathrm{VV}$ channel are displayed in Fig. 6 for the same number of looks of Fig. 2. First, comparing Fig. 6(a)-(b)-(c), we observe that noise reduction is more significant with larger multilook sizes, but the spatial resolution of the interferogram is clearly reduced. The NL-SAR filter is able to reduce the noise in some homogeneous areas, but noise remains high in very decorrelated zones. Concerning the polarimetric optimization, if we compare both rows in Fig. 6, some phase improvement is obtained with small multilook sizes and with the NL-SAR filter. This improvement is specially noticeable in areas that were not extremely decorrelated, such as in urban areas. The polarimetric optimization provides more homogeneous phase values in such areas, for which coherence was increased to a greater extent (see magnified subregions in the top of the images in Fig. 6). However, very decorrelated zones do not present any real improvement with regards to the conventional $\mathrm{HH}+\mathrm{VV}$ channel, as shown in the magnified subregions in the bottom of the images in Fig. 6. Additionally, for larger multilook sizes, the optimization does not provide a significant phase improvement with respect to channel $\mathrm{HH}+\mathrm{VV}$. If we compare Figs. 6(c) and (g) we observe that there is almost no difference between both channels, so that the noise reduction with respect to the original SLC data is mainly due to multilooking. Consequently, a trade-off between coherence improvement provided by polarimetric optimization and noise reduction by multilooking must be considered. Very noisy parts of an interferogram, as some analyzed in this work, would benefit from larger multilook sizes for a stronger noise reduction, but PolDInSAR algorithms would not be able to provide an additional phase improvement. On the contrary, polarimetric optimization methods can considerably increase the number of high-coherent pixels when smaller number of looks are employed. In these cases, the spatial smoothness of the phase can be improved in some localized areas, but noise reduction in wide decorrelated areas may not be enough.

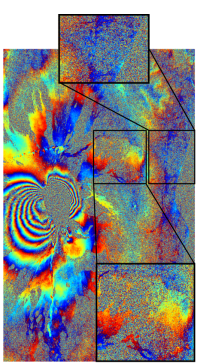

(a)

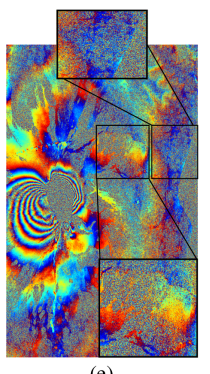

(e)

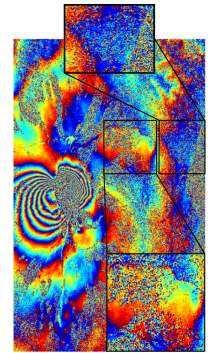

(b)

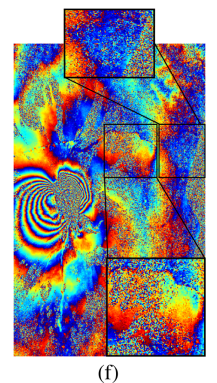

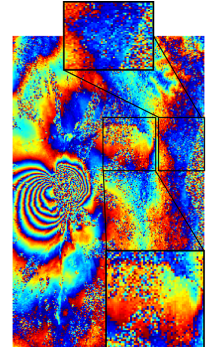

(c)

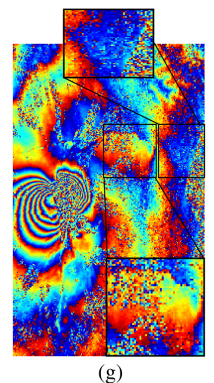

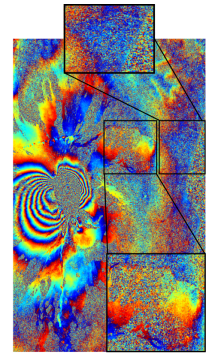

(d)

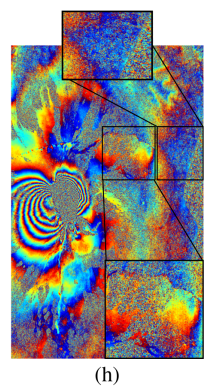

Fig. 6. Differential phases of channels $\mathrm{HH}+\mathrm{VV}$ (top) and computed optimum (bottom) for different number of looks: (a)-(e) $3 \times 3$. (b)-(f) $9 \times 9$. (c)(g) $19 \times 19$. (d)-(h) NL-SAR.

\section{B. Noise Reduction Analysis in Homogeneous areas}

A potential problem related to the optimization process we have employed lies in the algorithm itself, since it works on a pixel-by-pixel basis. Therefore, the optimum projection vector $\omega$ for two adjacent pixels can be different, even if they belong to the same kind of land cover. Then, the quality of the resulting phase may not be improved significantly, since different phase centers can be retrieved due to the selection of different polarimetric channels in neighbor pixels.

To study this issue, we have tested whether the spatial variability in the projection vectors is the reason of the small improvement in terms of noise reduction. To this end, the phase noise has been quantified with the phase standard deviation within homogeneous areas. Note that, for this study any residual phase gradient has been subtracted [14] in order to obtain true measurements of the phase standard deviation. This test is performed within the four different areas highlighted in Fig. 1. The same optimization has been carried out at fullresolution and with multilook sizes from $3 \times 3$ to $21 \times 21$, and with the NL-SAR filter. Unlike in the previous coherence optimization, a single optimum projection vector is computed for the whole ROI. 
TABLE I

COMPARISON OF THE PHASE STANDARD DEVIATION IN THE FOUR ANALYZED AREAS BETWEEN CHANNELS HH+VV AND THE OPTIMUM CHANNEL FOR DIFFERENT NUMBER OF LOOKS

\begin{tabular}{|c|c|c|c|c|c|}
\hline \multirow[b]{3}{*}{$\begin{array}{c}\text { Multilook } \\
\text { size }\end{array}$} & \multicolumn{5}{|c|}{ Phase standard deviation [rad] } \\
\hline & \multirow[b]{2}{*}{ Channel } & \multicolumn{3}{|c|}{ Area type } & \multirow[b]{2}{*}{ Changed } \\
\hline & & Rural & $\begin{array}{c}\text { With } \\
\text { vegetation }\end{array}$ & $\begin{array}{c}\text { Without } \\
\text { vegetation }\end{array}$ & \\
\hline SLC & $\begin{array}{l}\text { Optimum } \\
\mathrm{HH}+\mathrm{VV}\end{array}$ & $\begin{array}{l}1.645 \\
1.657\end{array}$ & $\begin{array}{l}1.762 \\
1.769\end{array}$ & $\begin{array}{l}0.721 \\
0.788\end{array}$ & $\begin{array}{l}1.786 \\
1.789\end{array}$ \\
\hline \multirow{2}{*}{$3 \times 3$} & Optimum & 1.451 & 1.704 & 0.302 & 1.762 \\
\hline & $\mathrm{HH}+\mathrm{VV}$ & 1.461 & 1.712 & 0.303 & 1.769 \\
\hline \multirow{2}{*}{$5 \times 5$} & Optimum & 1.275 & 1.652 & 0.271 & 1.738 \\
\hline & $\mathrm{HH}+\mathrm{VV}$ & 1.285 & 1.663 & 0.272 & 1.745 \\
\hline \multirow{2}{*}{$7 \times 7$} & Optimum & 1.110 & 1.597 & 0.259 & 1.708 \\
\hline & $\mathrm{HH}+\mathrm{VV}$ & 1.123 & 1.611 & 0.262 & 1.718 \\
\hline \multirow{2}{*}{$9 \times 9$} & Optimum & 0.961 & 1.539 & 0.252 & 1.695 \\
\hline & $\mathrm{HH}+\mathrm{VV}$ & 0.972 & 1.557 & 0.254 & 1.702 \\
\hline \multirow{2}{*}{$11 \times 11$} & Optimum & 0.829 & 1.481 & 0.247 & 1.670 \\
\hline & $\mathrm{HH}+\mathrm{VV}$ & 0.840 & 1.507 & 0.249 & 1.695 \\
\hline \multirow{2}{*}{$13 \times 13$} & Optimum & 0.721 & 1.423 & 0.242 & 1.653 \\
\hline & $\mathrm{HH}+\mathrm{VV}$ & 0.734 & 1.459 & 0.245 & 1.676 \\
\hline \multirow{2}{*}{$15 \times 15$} & Optimum & 0.621 & 1.365 & 0.238 & 1.627 \\
\hline & $\mathrm{HH}+\mathrm{VV}$ & 0.647 & 1.409 & 0.241 & 1.657 \\
\hline \multirow{2}{*}{$17 \times 17$} & Optimum & 0.539 & 1.299 & 0.235 & 1.601 \\
\hline & $\mathrm{HH}+\mathrm{VV}$ & 0.578 & 1.355 & 0.238 & 1.633 \\
\hline \multirow{2}{*}{$19 \times 19$} & Optimum & 0.475 & 1.262 & 0.231 & 1.580 \\
\hline & $\mathrm{HH}+\mathrm{VV}$ & 0.516 & 1.301 & 0.235 & 1.618 \\
\hline \multirow{2}{*}{$21 \times 21$} & Optimum & 0.425 & 1.221 & 0.228 & 1.569 \\
\hline & $\mathrm{HH}+\mathrm{VV}$ & 0.468 & 1.248 & 0.231 & 1.598 \\
\hline \multirow{2}{*}{ NL-SAR } & Optimum & 1.276 & 1.657 & 0.248 & 1.686 \\
\hline & $\mathrm{HH}+\mathrm{VV}$ & 1.293 & 1.669 & 0.251 & 1.729 \\
\hline
\end{tabular}

Results are summarized in Table I. For comparison purposes, we have also estimated the phase standard deviation of channel $\mathrm{HH}+\mathrm{VV}$. Vegetation and changed areas exhibit the highest values, showing an extreme decorrelation. The rural area has a slightly lower value, and finally the bare surface area is less affected by noise. Due to the multilook processing, the initial noise is reduced for all area types, so phase standard deviation values become lower as the multilook size increases, especially for the bare surface and the rural areas. However, if we compare the standard deviation of the optimum channel and the $\mathrm{HH}+\mathrm{VV}$ channel, there is not a significant improvement in noise reduction with regards to the conventional channel, so that noise suppression is mainly achieved with multilooking. Also, it is deduced that the pixel-by-pixel approach was not the reason for the minor improvement in noise reduction.

\section{CONCLUSiON}

We have analyzed the influence of the multilook size in PolDInSAR coherence optimization and phase improvement, although a single RADARSAT-2 interferogram relevant to the Mount Etna's volcanic eruption has been processed. For this data set, the largest improvement provided by polarimetry as been obtained with small multilook sizes and with the NLSAR filtered data. Phase noise is effectively reduced, obtaining more homogeneous phase values specially in low-vegetated areas or urban areas, and the number of high-coherent pixels is considerably larger than in conventional channels. However, very decorrelated areas benefit from larger multilook sizes, for which the polarimetric optimization does not provide a significant improvement with respect to conventional channels. In this kind of areas, noise reduction is mainly achieved by multilooking and there is an inability to find a polarimetric channel that minimizes the phase noise. In the next future we plan to evaluate polarimetric optimization methods with different data sets and to explore alternative ways to apply polarimetry to this problem. or instance, change detection techniques, based on polarimetry, can be employed to complement the interferometric coherence to evaluate the quality of the data in different areas. In addition, target decomposition could be used to guide phase filtering approaches in order to increase the number of looks over wide homogeneous areas (hence reducing the noise), while preserving small details.

\section{ACKNOWLEDGMENT}

RADARSAT-2 Data and Products (C)MacDonald, Dettwiler and Associates Ltd. (2008) - All Rights Reserved. RADARSAT is an official trademark of the Canadian Space Agency (CSA). All RADARSAT-2 images were provided by MDA and CSA in the framework of project SOAR 5087.

\section{REFERENCES}

[1] D. Massonnet, M. Rossi, C. Carmona, F. Adragna, G. Peltzer, K. Feigl, and T. Rabaute, "The displacement field of the Landers earthquake mapped by radar interferometry," Nature, vol. 364, pp. 138-142, July 1993.

[2] A. Hooper, H. Zebker, P. Segall, and B. Kampes, "A new method for measuring deformation on volcanoes and other natural terrains using InSAR persistent scatterers," Geophysical Research Letters, vol. 31, no. 23, 2004, 123611. [Online]. Available: http://dx.doi.org/10.1029/2004GL021737

[3] V. D. Navarro-Sanchez, J. M. Lopez-Sanchez, and F. Vicente-Guijalba, "A contribution of polarimetry to satellite differential SAR interferometry: Increasing the number of pixel candidates," IEEE Geoscience and Remote Sensing Letters, vol. 7, no. 2, pp. 276-280, April 2010.

[4] V. D. Navarro-Sanchez and J. M. Lopez-Sanchez, "Improvement of persistent-scatterer interferometry performance by means of a polarimetric optimization," IEEE Geoscience and Remote Sensing Letters, vol. 9, no. 4, pp. 609-613, July 2012.

[5] S. Alipour, K. F. Tiampo, S. Samsonov, and P. J. Gonzalez, "Multibaseline PolInSAR using RADARSAT-2 quad-pol data: Improvements in interferometric phase analysis," IEEE Geoscience and Remote Sensing Letters, vol. 10, no. 6, pp. 1280-1284, Nov 2013.

[6] V. D. Navarro-Sanchez, J. M. Lopez-Sanchez, and L. Ferro-Famil, "Polarimetric approaches for persistent scatterers interferometry," IEEE Trans. Geosci. Remote Sensing, vol. 52, no. 3, pp. 1667-1676, March 2014.

[7] R. Iglesias, D. Monells, X. Fabregas, J. J. Mallorqui, A. Aguasca, and C. Lopez-Martinez, "Phase quality optimization in polarimetric differential SAR interferometry," IEEE Trans. Geosci. Remote Sensing, vol. 52, no. 5, pp. 2875-2888, May 2014.

[8] R. Touzi, A. Lopes, J. Bruniquel, and P. W. Vachon, "Coherence estimation for SAR imagery," IEEE Trans. Geosci. Remote Sensing, vol. 37, no. 1, pp. 135-149, Jan 1999.

[9] J.-S. Lee, K. W. Hoppel, S. A. Mango, and A. R. Miller, "Intensity and phase statistics of multilook polarimetric and interferometric SAR imagery," IEEE Trans. Geosci. Remote Sensing, vol. 32, no. 5, pp. 10171028, Sept 1994.

[10] A. Monti Guarnieri and C. Prati, "SAR interferometry: a "quick and dirty" coherence estimator for data browsing," IEEE Transactions on Geoscience and Remote Sensing, vol. 35, no. 3, pp. 660-669, May 1997.

[11] C. A. Deledalle, L. Denis, F. Tupin, A. Reigber, and M. Jager, "NL-SAR: A unified nonlocal framework for resolution-preserving (Pol)(In)SAR denoising," IEEE Trans. Geosci. Remote Sensing, vol. 53, no. 4, pp. 2021-2038, April 2015.

[12] B. Wu, L. Tong, Y. Chen, and L. He, "Improved SNR optimum method in PolDInSAR coherence optimization," IEEE Geoscience and Remote Sensing Letters, vol. 13, no. 7, pp. 982-986, July 2016.

[13] S. R. Cloude and K. P. Papathanassiou, "Polarimetric SAR interferometry," IEEE Trans. Geosci. Remote Sensing, vol. 36, no. 5, pp. 1551-1565, Sep. 1998.

[14] Z. Suo, Z. Li, and Z. Bao, "A new strategy to estimate local fringe frequencies for InSAR phase noise reduction," IEEE Geoscience and Remote Sensing Letters, vol. 7, no. 4, pp. 771-775, Oct 2010. 